This item was submitted to Loughborough's Research Repository by the author.

Items in Figshare are protected by copyright, with all rights reserved, unless otherwise indicated.

\title{
Reliability modelling of automated guided vehicles using Petri nets
}

\section{PLEASE CITE THE PUBLISHED VERSION}

https://www.crcpress.com/Risk-Reliability-and-Safety-Innovating-Theory-and-Practice-Proceedings/WallsRevie-Bedford/p/book/9781138029972

\section{PUBLISHER}

(C) Taylor \& Francis

\section{VERSION}

AM (Accepted Manuscript)

\section{PUBLISHER STATEMENT}

This work is made available according to the conditions of the Creative Commons Attribution-NonCommercialNoDerivatives 4.0 International (CC BY-NC-ND 4.0) licence. Full details of this licence are available at: https://creativecommons.org/licenses/by-nc-nd/4.0/

\section{LICENCE}

CC BY-NC-ND 4.0

\section{REPOSITORY RECORD}

Yan, Rundong (Derek), Sarah Dunnett, and Lisa Jackson. 2016. "Reliability Modelling of Automated Guided Vehicles Using Petri Nets". figshare. https://hdl.handle.net/2134/24575. 


\title{
Reliability modelling of automated guided vehicles using Petri nets
}

\author{
R.D. Yan, S.J. Dunnett, L.M. Jackson \\ Loughborough University. Loughborough, Leics., U.K.
}

\begin{abstract}
Automated guided vehicles (AGVs) are being extensively used due to their attributions of high efficiency and low costs. To assure their added value, taking a typical AGV transport system as an example, the reliability issues in AGVs are investigated in this paper. First of all, the AGV transport system was modelled as a phased mission that comprises a few key phases. Then, the Petri net (PN) method is applied to describe the logic of the whole phase mission and based on this, the reliability of the mission is assessed via Monte-Carlo simulation. In order to validate the reliability assessment result by the PN method, the theoretical reliability of the AGV system is also assessed through performing fault tree analysis (FTA). The comparison indicates that both methods give very similar results. Thus, it can be concluded that apart from FTA, the PNs method is also a reliable tool for AGV system reliability assessment.
\end{abstract}

\section{INTRODUCTION}

Since being introduced in 1955 (Müller, 1983), Automated Guided Vehicles (AGVs) are being increasingly used today for intelligent transportation and distribution of materials in warehouses and/or manufacturing facilities. Such vehicles travel along predefined routes to deliver various tasks without the supervision of an on-board operator. Their reliability is crucial for ensuring normal industry production. However, the investigation and research about the reliability of AGVs are very few.

From the openly published literature, it is known that Fazlollahtabar (2013) created a model in order to maximize the reliability of AGVs and minimize their repair cost. However, there are some fundamental questions, such as 'How could AGVs fail?' and 'What are the possibilities of their failure?', which were not yet answered in their work. To answer these questions, Duran et al. (2013) has tried to identify the basic failure modes of the light detection and ranging (LIDAR) system and the camera-based computer vision system (CV) on AGVs by using a combined approach of Fault Tree Analysis (FTA) and Bayesian Belief Networks (BN). In the work, human injury, property damage and vehicle damage were defined as the top events in the fault tree. However, the research did not cover all components and subassemblies included in AGVs. Considering a complete investigation of the reliability issues of all AGV components and subassemblies is fairly impor- tant not only to ensure the high reliability and availability of AGVs and their success of delivering prescribed tasks but also to optimize their maintenance strategies. The reliability issues of the whole AGV systems are investigated through assessing the reliability of a typical AGV transport system in this paper.

In order to identify the crucial mission phases in AGV operations and predict the reliability of the whole AGV system, Petri net (PN) simulation is applied to the reliability assessment of the AGV. To ensure the correctness of the PN method, fault tree analysis (FTA) is also performed for the purpose of validation.

The remaining part of the paper is organized as follows. In Section 2, a typical AGV system and their subassemblies are briefly introduced; in Section 3 , the basic principles of the PN modelling method are explained; in Section 4, reliability analysis of a typical AGV transport system is performed by using the PN method. Where, the fault trees for all phases, particularly phases 1 and 2, are given in order to establish the logic that is essential for PN simulation; in Section 5, the mission reliability at the end of each phase and the unreliability of each phase of a typical AGV transport system are calculated by using the PN method. Moreover, the PN method is verified through comparing its calculation results with those obtained from FTA; the paper is finally concluded in Section 6. 


\section{BRIEF DESCRIPTION OF A TYPICAL AGV SYSTEM}

In this paper, to facilitate the research a typical AGV transport system used in a warehouse for material distribution is chosen for analysis. As shown in Figure 1, the AGV system consists of the following components:

(1) drive unit - a brushless DC electric motor which is responsible to provide power for the motion and operation of the AGV;

(2) software control system - is responsible to process and interpret the information received from both the laser navigation system and safety system, and send either motion or operation orders;

(3) laser navigation system - was developed by MacLeod et al. (1993). In the AGV, it is in essence a position measurement system that is responsible to locate the AGV. It comprises a rotating laser installed on the board of the AGV and three beacons mounted along the border of the area covered in a warehouse;

(4) safety system - is designed to avoid obstacles that could appear on the pathway with the aid of a laser detection system installed on the AGV;

(5) attachments - refers to those additional components that are used to assist moving and carrying of items;

(6) batteries - are usually the common lead-acid batteries. They are responsible to supply power to the whole AGV system;

(7) brake system - is responsible to slow down or stop the AGV in the case of necessities. It is always applied when the AGV stands still in parking space;

(8) steering system - is responsible to dominate the manoeuvre of the AGV;

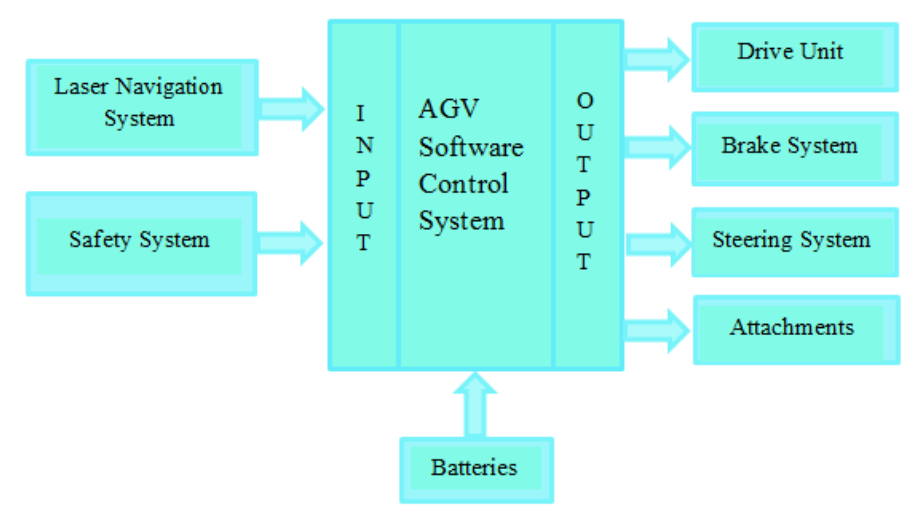

Figure 1. A typical AGV system schematic.

If all these aforementioned components are normal in health, then their synchronous operation will enable the AGV to successfully distribute materials to multiple places in a warehouse according to different requirements. But when receiving a task order, the AGV will, first of all, optimize the routes for completing the whole mission. The routes that will be optimized include:

(1) the route from its parking position to material collection port;

(2) the route from material collection port to target position; and

(3) the route from target position back to its original parking position.

Initially, the AGV will travel to the material collection port along the optimized route to pick up the materials. After the AGV is loaded with the materials, it will travel to the destination and unload the materials. After successfully distributing the materials, the AGV will travel back to its original parking position. Therefore, the whole mission can be divided into 6 phases in total, namely mission allocation and route optimization, dispatch to station, loading of item, travelling to storage, unloading and finally travelling back to base. The mission can be regarded as successful only when the AGV is able to operate successfully throughout all these 6 phases without any break due to component and/or system failures and maintenance. Such a period is named as a maintenance-free operational period (MFOP) (Division, 1998).

\section{RELIABILITY MODELLING METHODS}

In the past decades, FTA has been widely adopted for modelling the reliability issues met in industrial practice. Through inspecting the logic between the undesired events that could happen in a system or a mission, FTA allows us to trace back the root cause of a system or mission failure by using a systematic top-down approach. Moreover, with the aid of FTA the probability of system or mission failure can be computed via Boolean logic calculations. Attributed to that FTA provides a straightforward and clear presentation to the logic between various undesired events and moreover it supports both qualitative and quantitative analyses. FTA has been regarded as an effective, systematic, accurate and predictive method to deal with the safety and reliability problems in complex systems, such as the safety issues in a nuclear power plant (NUREG/1150, 1990). However, it is difficult to construct a fault tree when the system of interest is large and complex. For this reason, an alternative reliability modelling method, namely PNs, was developed by Petri in 1962 (Petri, 1962).

Similar to FTA, PN provides an intuitive graphical representation of the reliability problem being investigated. But by contrast, the PN method is more suited to dealing with the reliability issues in complex systems attributed to their efficient computing algorithm. 
The PNs method is, in essence, a direct bipartite graph. As shown in Figure 2, it basically consists of the following four types of symbols:

- Circles - represent the places, which are conditions or states such as mission failure, phase failure, or component failure depending on the issue being considered;

- Rectangles - represent the transitions, more abstractly actions or events. It should be mentioned that if time for completing the transition is zero, a solid rectangular bar can be used, otherwise it is hollow;

- Arrows - represent connections between places and transitions. It should be noticed that arcs with one slash on it and a number next to it represent a combination of several single arcs with a weight to/from the same transition. No slash always means that the weight is one;

- Small marks - represent tokens which carry the information in PNs. They move via transitions as long as the enabling condition is satisfied, which gives dynamic properties of the PN.

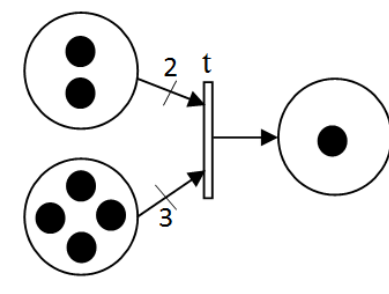

(a)

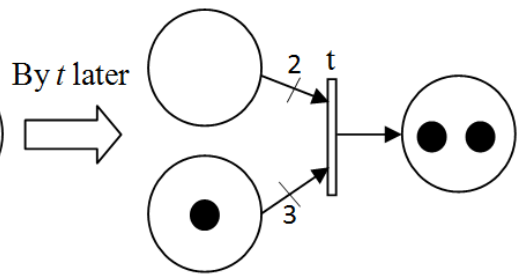

(b)
Figure 2. Enabling and switching of transition, (a) before enabling transition, (b) after enabling transition.

Figure 2 shows an example of the movement of tokens through a net. From Figure $2 a$, it is seen that there are two inputs and one output place connected to a timed transition with a time delay $t$. The input places have arcs with weights 2 and 3 , respectively. The transition is enabled when the number of tokens contained in every input place is not less than the corresponding arc weights. Once the transition is enabled, the arc weight number of tokens will be taken out from the corresponding input place to fulfil the transition after the time delay $t$ associated with the transition. For the example shown in figure 2 one more token will appear in the output place. But it is necessary to note that after completing the transition, the number of tokens that are increased in the output place is dependent on the corresponding arc weight as well. For example, if the arc weight connected to the output place is ' $n$ ', then $n$ more tokens will appear in the output place after enabling the transition.

In the aspect of phase mission simulation, Mura \& Bondavalli (2001) proposed to use two distinct PNs, namely a system net and a phase net, to model phased missions; Such an idea was later further ex- tended by Chew et al. (2008) to simulate more complex systems by using three distinct PNs, i.e. phase PN (PPN), component PN (CPN) and master PN (MPN). These three kinds of PNs are linked together and interact with each other. Such an extended approach is adopted in this paper to assess the reliability of AGV systems.

\section{RELIABILITY ANALYSIS OF AN AGV TRANSPORT SYSTEM USING THE PN METHOD}

In this section, the reliability problem of a typical AGV transport system will be investigated by using the extended PN-based method mentioned in section 3 . The details are depicted as follows.

\subsection{Phase Petri net}

The PPN presents the system failure at each phase due to component failures. The logic used for implementing FTA can be directly applied for constructing PPN. For this reason, the fault trees constructed for phases 1 and 2 are shown in Figure 3 to ease understanding.

From Figure 3, it is seen that in each phase level fault tree, the failure of phase mission is used as the top event, the failures of those AGV components that are involved in the phase are basic events. The failures of mechanical parts, the failures of system parts for navigation, control and safety, and the failures of power supply are the intermediate events. From these fault trees, how the identified AGV components affect the success of each phase has been clearly presented. For example, in the first phase 'mission allocation and route optimisation', first of all the laser navigation system (LNS) needs to correctly locate the AGV's position. Then, all routes for completing the phase mission should be properly optimised via the AGV software control system (ASCS). Once either one of LNS or ASCS fails to work properly, the phase 1 mission cannot be accomplished. Moreover, the successful completion of phase 1 mission also relies on safe power supply, the failure of which can lead to unsuccessful phase 1 as well. In Phase 2, the AGV will travel from its parking position to the material collection port. During the period, the ASCS will control the AGV to travel along the optimised route; the LNS works over the whole course of the phase to locate the $\mathrm{AGV}$ as it moves; the motor is required to drive the vehicle; the steering system enables vehicle turning; the safety system performs obstacle scan; and the brake system is responsible to slow down the vehicle when turning and stop the vehicle to avoid collisions. Obviously, the success of phase 2 mission relies on the synchronously cooperation of all these subassemblies. The fault of either one of them can 
lead to the failure of phase 2. In addition, it is worthy to note that phase 2 can be started only after phase 1 has been completed successfully. In other words, the mission failure in phase $j+1$ is the combined result of successful phases 1 to $j$ and the system failure occurring in phase $j+1$ via an 'AND' gate. In addition, in Figure 3 the 'NOT' gate is used to represent system success during a phase. Following this logic, the AGV operation is analysed at each phase.

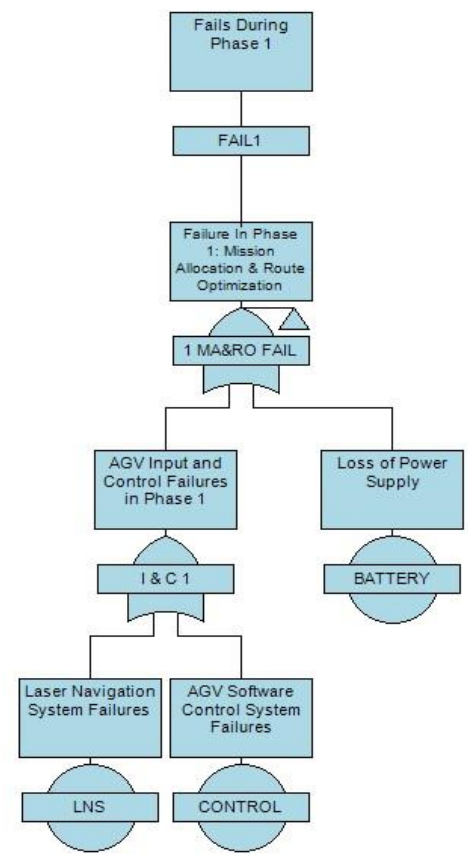

(a)

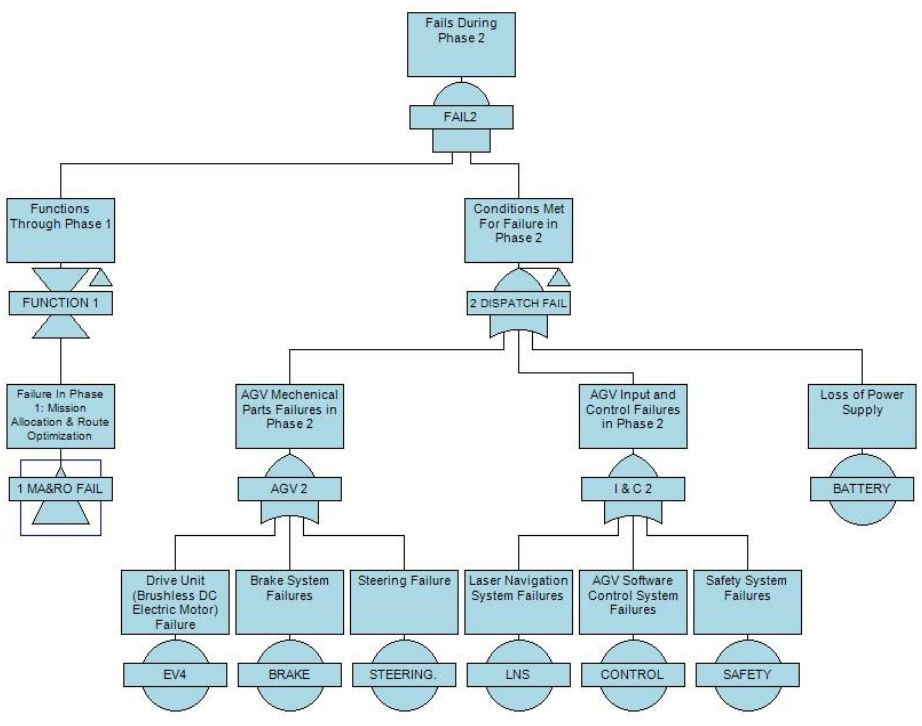

(b)

Figure 3. Fault trees for Phases 1 and 2, (a) Phase 1, (b) Phase 2.

Likewise, the logic existing in phases 3-6 can be readily identified as well. Herein, they will not be described to keep a concise context. But to facilitate analysis, the component failures that lead to the system failure at different phases are listed in Table 1, from which the logic in each phase can be inferred.
Table 1: Component failures that cause the system failure at each phase.

\begin{tabular}{ll}
\hline Phase & $\begin{array}{l}\text { Component failures causing system failure at } \\
\text { each phase }\end{array}$ \\
\hline 1 & ASCS; LNS; Batteries \\
2 & $\begin{array}{l}\text { Drive unit (DC); Brake system; Steering system; } \\
\text { ASCS; LNS; Safety system (SS); Batteries }\end{array}$ \\
& $\begin{array}{l}\text { Attachments; Brake system; ASCS; } \\
\text { Safety system; Batteries; }\end{array}$ \\
& Drive unit; ASCS; LNS; Safety system (SS); \\
& Attachments; Batteries; Brake system; \\
& Steering system \\
& Attachments; Brake system; ASCS; \\
5 & Safety system (SS); Batteries; \\
& Drive unit; ASCS; LNS; Safety system(SS); \\
6 & Batteries; Brake system; Steering system \\
\hline
\end{tabular}

Once the logic existing at all phases are identified, the PPN at each phase can be constructed. The PPN corresponding to the fault trees depicted in Figure 3 are shown in Figure 4. Where 'P', 'IC' and 'MP' stand for phase, input and control in the phase, and mechanical part in the phase, respectively. In addition, 'tokens' are absent from all places in Figure 4. That means the whole AGV system and its components are in the good health condition. In other words, the presence of a token in a place will mean the failure of either a component or a phase.

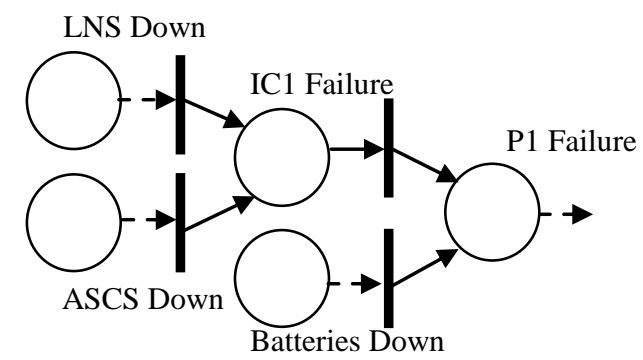

(a)

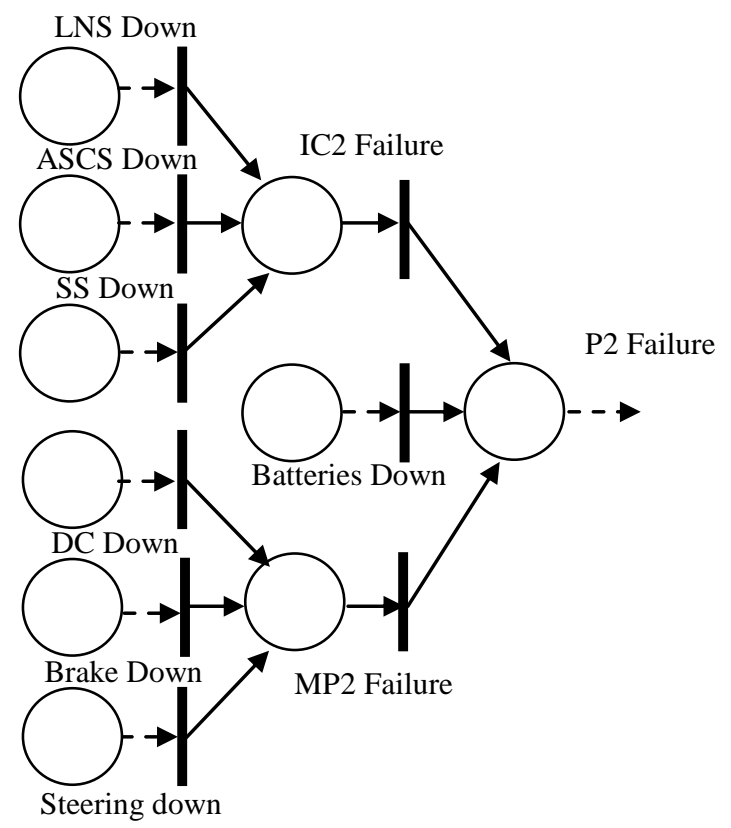

(b)

Figure 4. Phase Petri net, (a) for phase 1, (b) for phase 2. 
From Figure 4, it is noticed that the transition is symbolized by solid rectangular bars. That implies a component failure will result in a system failure instantly without any time delay.

\subsection{Component Petri Net}

As shown in Figure 5, the CPN is used to model the health states of AGV components. As the mission has been modelled as a MFOP, the repair of components will not be considered in this study. Therefore, the components in the CPN will show only two kinds of health states, i.e. 'normal' and 'abnormal'. Once a component fails after working for a certain period of time, the token in the 'component up' places will be transferred to the 'component down' places. The time for this failure transition can be computed by using the random sampling and exponential distribution method, which is supported by the component failure rate data (Andrews \& Moss, 2002). For simplicity, in this paper all of the AGV components are assumed to be independent of each other, which means that the failure rate of a certain component will not be affected by other AGV components. The information about component failures can then be fed into different PPN using linkingarcs, which are the dashed lines in Figure 5.

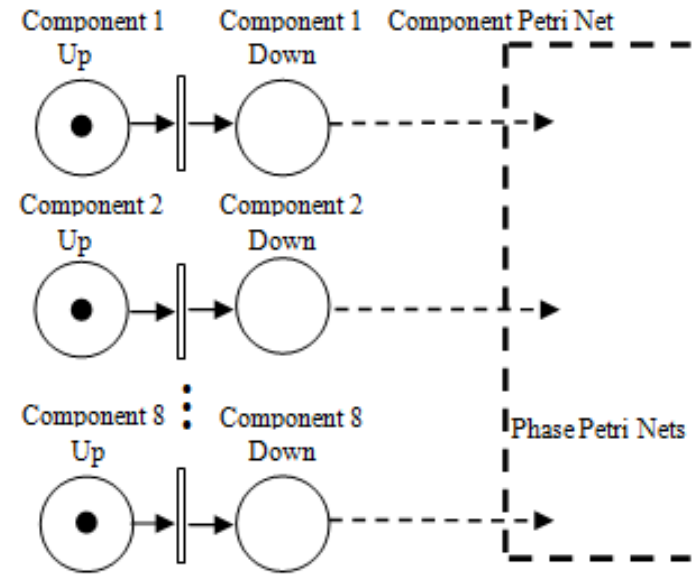

Figure 5. Component Petri Net.

\subsection{Master Petri net}

As Figure 6 shows, the MPN is used to govern the change of phases from the beginning of Phase 1 to the successful completion of the whole mission. Herein, the token in phase place is used to indicate the phase that the AGV is operating at. The system failure happening at each phase, i.e. the top event of the PPN for each phase, will directly result in the failure of the whole mission. Hence, the token in the phase mission flow will be transferred to the system failure place, so that the mission fails. The switching time of transition between two neighbouring phase places is the length of the first phase. Likewise, the switching time of transition between phase 6 and mission finished is the length of phase 6 .

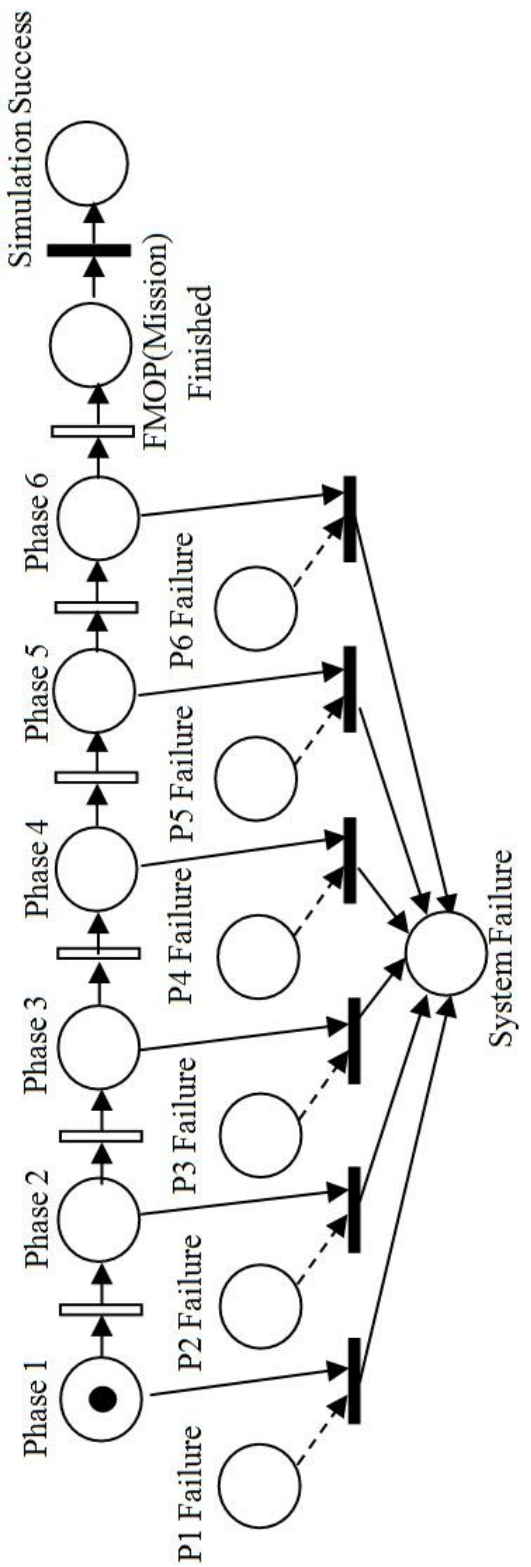

Figure 6. Master petri net.

\subsection{Simulation Model}

The failure rates of all AGV components mentioned in Section 4.2 and the phase lengths in Section 4.3 are used as inputs of PN simulation. Then, the simulation can be programmed by using the following steps based on the logic established above.

Step 1: Import the phase lengths into the MPN and in parallel, generate the switching time of the transitions of each component in the CPN's by using the randomly sampling and exponential distribution method;

Step 2: Find the transition with the minimum switching time and then switch it;

Step 3: Search through the immediate transitions that are directly connected to the present place. 
If any are found enabled, switch them;

Step 4: Repeat Step 3 until no more immediate transitions are enabled;

Step 5: Test for any of the following conditions and log them:

(a) if system has failed, begin next simulation;

(b) if mission has completed, begin next simulation.

Step 6: Iterate the above simulation for $n$ times based on the assumption that the reliability of the AGV system can be obtained by repeating the simulation for a sufficient number of times.

\section{RESULTS AND DISCUSSION}

In order to implement the calculation of the mission reliability at the end of each phase and the assessment of the unreliability of each phase, the information of the length of phase (i.e. the time duration for completing each phase) and component failure rate is listed in Tables 2 and 3, respectively. The total time duration to complete the whole mission is 0.51 hours.

Herein, it is worthy to note that all data listed in Tables 2 and 3 are empirical data only for demonstration purpose. In reality, they would be different when the $\mathrm{AGV}$ is requested to deliver different types of missions.

Table 2: Assumed phase lengths.

\begin{tabular}{ll}
\hline Phase & Phase Length \\
\cline { 2 - 2 } & hour \\
\hline Mission Allocation \& Route Optimization & 0.02 \\
Dispatch to Station & 0.2 \\
Loading of Item & 0.02 \\
Travelling to Storage & 0.15 \\
Unloading & 0.02 \\
Travelling Back to Base & 0.10 \\
\hline
\end{tabular}

Table 3: Assumed component failure rates.

\begin{tabular}{ll}
\hline Identity & Failure Rate \\
\cline { 2 - 2 } & frequency/year \\
\hline Drive Unit & 1.5 \\
AGV Software Control System & 6 \\
Laser Navigation System & 0.875 \\
Safety Systems & 0.375 \\
Attachments & 2 \\
Batteries & 6 \\
Brake System & 2 \\
Steering System & 4 \\
Manual button & 2 \\
\hline
\end{tabular}

The data in Tables 2 and 3 is applied as described in Section 4 to calculate the unreliability of each phase and the mission reliability at the end of each phase. The obtained calculation results are shown in Table 4. In order to ensure a good convergence of the computing result, one billion simulations are performed in the process of this calculation.

Table 4: PN simulation results.

\begin{tabular}{lllll}
\hline Phase & $\begin{array}{l}\text { Phase } \\
\text { failures }\end{array}$ & $\begin{array}{l}\text { Phases } \\
\text { started }\end{array}$ & $\begin{array}{l}\text { Phase } \\
\text { unreliability }\end{array}$ & $\begin{array}{l}\text { Mission } \\
\text { reliability at } \\
\text { phase end }\end{array}$ \\
\hline 1 & 18449 & 1000000000 & 0.00001845 & 0.999982 \\
2 & 244863 & 999981551 & 0.00024486 & 0.999737 \\
3 & 72843 & 999736688 & 0.00007286 & 0.999664 \\
4 & 218911 & 999663845 & 0.00021898 & 0.999445 \\
5 & 22488 & 999444934 & 0.00002250 & 0.999422 \\
6 & 125509 & 999422446 & 0.00012558 & 0.999297 \\
\hline
\end{tabular}

From the calculation results presented in Table 4, it is found that phase 2 'dispatch to station' and phase 4 'travelling to storage' show the largest phase unreliability values. This means that the AGV is more likely to fail when it delivers the tasks of these two phases. Additionally, it is noticed that as imagined, the mission reliability at the end of each phase decreases gradually against the number of phases that the AGV has successfully completed. This suggests that without maintenance, the more missions are completed, the more unreliable the AGV system will tend to be.

In order to validate the aforementioned PN method and the reliability of the mission assessment results obtained from it, FTA is also performed in this paper for verification purposes. The details of FTA are introduced as follows.

Firstly, the system failure in phase $j$, i.e. $T_{j}$, is calculated by using the following equation

$T_{j}=($ Phase 1 to j-1 Success $) \cdot($ Phase j Failure $)$

Then, the probability of failure of basic event $A$ in all phases from $i$ to $j$ (i.e. $q_{A i, j}$ ) can be calculated using the equation

$q_{A_{i, j}}=e^{-\lambda_{A} t_{i-1}}-e^{-\lambda_{A} t_{j}}$

where $\lambda_{A}$ refers to the failure rate of a basic event $A$, $t_{j}$ is the length of phase $j$.

The unreliability of phase $j$ can be calculated by $Q_{j}=1-R_{j}=1-\frac{R_{1, j}}{R_{1, j-1}}$

where $R_{j}$ denotes the success probability of phase $j$, $R_{l, j}$ is the success probability till the end of phase $j$.

In the FTA calculation, the component will be taken into account only when it is involved to complete a phase. It will not be considered if it is irrelevant to the phase. Applying the aforementioned method to calculate the unreliability of each phase and the mission reliability at the end of each phase within MFOP, the obtained results are listed in Table 5. 
Table 5: The resultant mission reliability and phase unreliability.

\begin{tabular}{lll}
\hline Phase & Phase unreliability & $\begin{array}{l}\text { Mission reliability at phase } \\
\text { end }\end{array}$ \\
\hline 1 & 0.00001855 & 0.999981 \\
2 & 0.00024386 & 0.999738 \\
3 & 0.00007266 & 0.999665 \\
4 & 0.00021915 & 0.999446 \\
5 & 0.00002243 & 0.999423 \\
6 & 0.00012527 & 0.999298 \\
\hline
\end{tabular}

The comparison of Tables 4 and 5 shows that the simulation results obtained from PN method are very close to those analytical solutions derived from FTA. The simulation errors of both the unreliability of each phase and the mission reliability at the end of each phase are below $1 \%$. To ease understanding, the details of the comparison of phase unreliability results are given in Table 6. This fully demonstrates that the PN method is as accurate as FTA in AGV reliability assessment. Thus, it is a promising time saving and cost-effective approach that can be widely used in the future to deal with the reliability problems existing in complex systems.

Table 6. Comparison of analytical and simulation unreliability results.

\begin{tabular}{llll}
\hline Phase & FTA Analysis & PN Simulation & Average error \\
\cline { 4 - 4 } & & & $\%$ \\
\hline 1 & 0.00001855 & 0.00001845 & 0.545 \\
2 & 0.00024386 & 0.00024486 & 0.412 \\
3 & 0.00007266 & 0.00007286 & 0.282 \\
4 & 0.00021915 & 0.00021898 & 0.077 \\
5 & 0.00002243 & 0.0000225 & 0.309 \\
6 & 0.00012527 & 0.00012558 & 0.243 \\
\hline
\end{tabular}

In addition, in order to demonstrate that one billion simulations are good enough to ensure a reliable assessment result, the variation tendency of the convergence curve when calculating Phase 1 unreliability is shown in Figure 7.

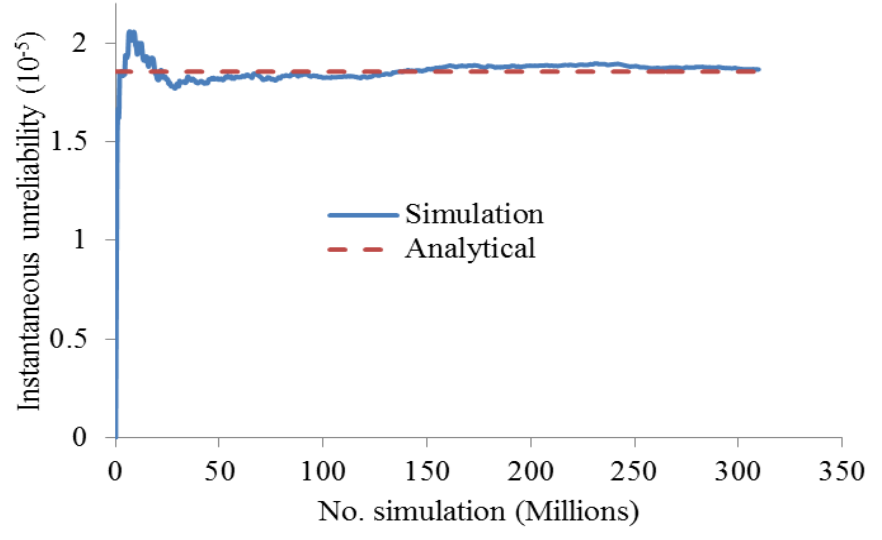

Figure 7. Convergence of phase 1 unreliability.

From Fig.7, it can be found that the value of unreliability of phase 1 has converged to the analytical result after performing 100 million simulations. So, performing 1 billion simulations in this paper is suf- ficient enough to guarantee the reliability of the calculation result. Thus, all PN calculation results and the conclusions drawn from them are trustable.

\section{CONCLUSIONS}

In order to develop an efficient and reliable approach to assessing the reliability of AGVs, PN method is tried in this paper to calculate the mission reliability at the end of each phase and assess the unreliability of each phase of a typical AGV transport system. Moreover, the calculation results are verified by the FTA. Through this research, the following conclusions can be drawn:

(1) The results obtained by PN model are very close to these obtained from FTA. This fully demonstrates that PN method is an effective approach to conducting system reliability assessment;

(2) Both PN simulation and FTA calculation have suggested that the AGV is more likely to fail when completing the phase 'dispatch to station' and the phase 'travelling to storage'. But it is worthy to note that such a judgement is made only based on the assumptions given in Tables 2 and 3 . In reality, the judgement result would be different, depending on the environmental, loading and operational conditions of the AGVs;

(3) Both FTA and PNs calculation results suggest that if without maintenance, the more missions are completed, the more unreliable the AGV system will tend to be;

(4) In contrast to FTA, PN method provides a more convenient approach to predicting the reliability of complex systems since the PN method does not rely on the calculation of analytical equations, which are often difficult to establish, particulalrly for complex systems. Also the PN approach is able to account for dependencies which may occur, which FTA cannot do.

\section{Acknowledgement}

The work reported in this paper is supported by EPSRC. The authors would like to extend thanks to Dr Cunjia Liu at Loughborough University and $\mathrm{Mr}$ Dave Berridge at Automated Materials Handling Systems Association for their kind help in preparing this paper.

\section{References}

Andrews J.D. \& Moss T.R. 2002. Reliability and Risk Assessment (second Edition), Professional Engineering 
Publishing.

Chew S.P., Dunnett S.J. \& Andrews J.D. 2008. Phased mission modelling of systems with maintenance-free operating periods using simulated Petri nets, Reliability Engineering and System Safety 93: 980-994.

Division M. 1998. Design for success Ultra Reliable Aircraft project, Design 212: 371-378.

Duran D.R., Robinson E., Kornecki A.J. \& Zalewski J. 2013. Safety Analysis of Autonomous Ground Vehicle Optical Systems: Bayesian Belief Networks Approach, Computer Science and Information Systems (FedCSIS), Federated Conference.

Fazlollahtabar H. \& Saidi-Mehrabad M. 2013. Optimising a muti-objective reliability assessment in multiple AGV manufacturing system, International journal of services and operations management 3: 352-372.

MacLeod E.N. \& Chiarella M. 1993. Navigation and control breakthrough for automated mobility, Proceedings of the SPIE mobile robotics VIII, Boston MA.

Mura I. \& Bondavalli A. 2001. Markov Regenerative Stochastic Petri Nets to Model and Evaluate Phased Mission Systems Dependability, IEEE Trans Comput 50(12): 1337-1351.

Müller T. 1983. Automated Guided Vehicles. IFS (Publications) Ltd./Springer-Verlag, UK/Berlin.

NUREG/1150, 1990. Severe Accident Risks: An Assessment for Five U.S. Nuclear Power Plants — Final Summary Report, Office of Nuclear Regulatory Cesearch 1.

Petri C A. 1962. Kommunikation mit automaten, PhD thesis. 